\title{
Fission fragment mass and total kinetic energy distributions of spontaneously fissioning plutonium isotopes
}

\author{
K. Pomorski ${ }^{\star}$, B. Nerlo-Pomorska, J. Bartel**, and C. Schmitt*,**** \\ UMCS, Lublin, Poland, ${ }^{* *} I P H C$, Strasbourg, France, ${ }^{* * *}$ GANIL, Caen, France
}

\begin{abstract}
The fission-fragment mass and total kinetic energy (TKE) distributions are evaluated in a quantum mechanical framework using elongation, mass asymmetry, neck degree of freedom as the relevant collective parameters in the Fourier shape parametrization recently developed by us. The potential energy surfaces (PES) are calculated within the macroscopic-microscopic model based on the Lublin-Strasbourg Drop (LSD), the Yukawa-folded (YF) single-particle potential and a monopole pairing force. The PES are presented and analysed in detail for even-even Plutonium isotopes with $A=236-246$. They reveal deep asymmetric valleys. The fission-fragment mass and TKE distributions are obtained from the ground state of a collective Hamiltonian computed within the BornOppenheimer approximation, in the WKB approach by introducing a neck-dependent fission probability. The calculated mass and total kinetic energy distributions are found in good agreement with the data.
\end{abstract}

\section{Introduction}

Our present understanding of nuclear fission is still based on the idea of Lisa Meitner and Otto Frisch [1] of a deformed charged liquid drop. In a seminal paper [2] Niels Bohr and John A. Wheeler fully developed the concept of the energy surface of a nucleus as function of a set of deformation parameters. The height of the minimal energy barrier that the nucleus has to overcome in the multidimensional energy hyper-surface as function of the deformation parameters on its decay path determines the stability of the nucleus against fission. Unfortunately, following the Bohr-Wheeler paper, one sometimes still uses up to now the Lord Rayleigh expansion of the nuclear surface into spherical harmonics. It was shown (e.g. in Ref. [3]) that such an expansion is not rapidly converging for large deformations and one needs to include terms up to multipolarity 16 in order to describe shapes close to the scission point. Fortunately, some better parametrizations of nuclear shapes exist. Among the most popular are the Quadratic Surfaces of Revolution (QSR) originally proposed by Nix [4], the Funny-Hills parametrization and its extension [5, 6], and the Cassini ovals with modifications due to Pashkevich [7]. The first two of the above quoted parametrizations are not analytical and closed (e.g. do no allow for the inclusion of higher order terms) while the last one is not easy to handle and its parameters do not have a clear physical interpretation.

The recently developed Fourier parametrization (see Appendix) of deformed nuclear shapes $[8,9]$ is free of these ambiguities and is rapidly converging. Using this parametrisation we have made an

^e-mail: Krzysztof.Pomorski@umcs.pl 
attempt to obtain the fission-fragment mass and total kinetic energy distribution of even-even Plutonium isotopes with mass numbers $236 \leq A \leq 246$. The deformation-energy landscapes that we are going to discuss in Sect. 4 are described by three Fourier deformation parameters $q_{2}, q_{3}, q_{4}$ which are respectively elongation, mass-asymmetry and neck degree of freedom of the fissioning nucleus $[8,9]$. Nonaxial shapes were not included in the present research since at large nuclear deformations they turn out to play a minor role. Thanks the fast convergence of the Fourier series, we believe that the above defined three deformation parameters are sufficient to describe the large variety of nuclear shapes up to very large deformations [9].The potential energy surfaces of different fissioning nuclei were calculated within the macroscopic-microscopic method $[5,10]$. The fragment mass distribution obtained in low-energy fission of light actinides was evaluated in a quantum mechanics framework within the Born-Oppenheimer approximation (BOA) [11].

Within the aforementioned deformation space the fission yield is obtained from the probability distribution of the collective wave function on the $\left(q_{3}, q_{4}\right)$ plane in the vicinity of the scission configuration $\left(q_{2} \approx 2.3\right)$. A neck-size dependent fission probability [12] was used to evaluate the fissionfragment mass and kinetic energy yields from the distribution probability at different elongations of the fissioning nucleus as will be explained in Sect. 3.

\section{Macroscopic-microscopic model of the potential energy}

The nuclear deformation energies of our analysis were determined in the macroscopic-microscopic approach, where the Lublin Strasbourg Drop (LSD) model [13] has been used for the macroscopic part of the potential-energy surface. Microscopic effects have been evaluated through a Yukawafolded (YF) single-particle potential [14] with the parameters listed in Ref. [15], where also our way of solving of the eigenproblem of the YF Hamiltonian is described. Eighteen deformed harmonic oscillator shells were taken into account when diagonalising the YF Hamiltonian. The Strutinsky shell-correction method $[5,16]$ with a $8^{\text {th }}$ order correctional polynomial and a smearing width $\gamma_{S}=$ $1.2 \hbar \omega_{0}$ is used, where $\hbar \omega_{0}=41 / A^{1 / 3} \mathrm{MeV}$ is the spherical harmonic-oscillator frequency. The BCS [10] theory, including an approximate GCM+GOA particle-number projection as described in Refs. [17] was used for the pairing correlations. The pairing strength equal to $G \cdot \mathcal{N}^{2 / 3}=0.28 \hbar \omega_{0}$, (with $\mathcal{N}=Z, N$ for protons or neutrons) was adjusted to the experimental mass differences of nuclei in this region using a pairing window composed of $2 \sqrt{15 \mathcal{N}}$ single-particle levels closest to the Fermi surface [18].

\section{Collective model of fission}

In any coordinates $q_{n}$ the collective Hamiltonian has the following form:

$$
\widehat{H}_{\text {coll }}=-\frac{\hbar^{2}}{2} \sum_{i, j}|M|^{-1 / 2} \frac{\partial}{\partial q_{i}}|M|^{-1 / 2} M_{i j}^{-1} \frac{\partial}{\partial q_{j}}+V,
$$

where $M_{i j}\left(\left\{q_{i}\right\}\right)$ and $V\left(\left\{q_{i}\right\}\right)$ denote the inertia tensor and the potential energy, respectively and $|M|=$ $\operatorname{det}\left(M_{i j}\right)$.

The eigenproblem of this Hamiltonian can be solved in the BOA in which one assumes that the motion towards fission, i.e. in the $q_{2}$ coordinate, is much slower than the motion in the $q_{3}$ and $q_{4}$ collective variables. This implies that the eigenfunction of $\widehat{H}_{\text {coll }}$ can be approximated in our case in the following form:

$$
\Psi_{n E}\left(q_{2}, q_{3}, q_{4}\right)=u_{n E}\left(q_{2}\right) \varphi_{n}\left(q_{3}, q_{4} ; q_{2}\right) .
$$



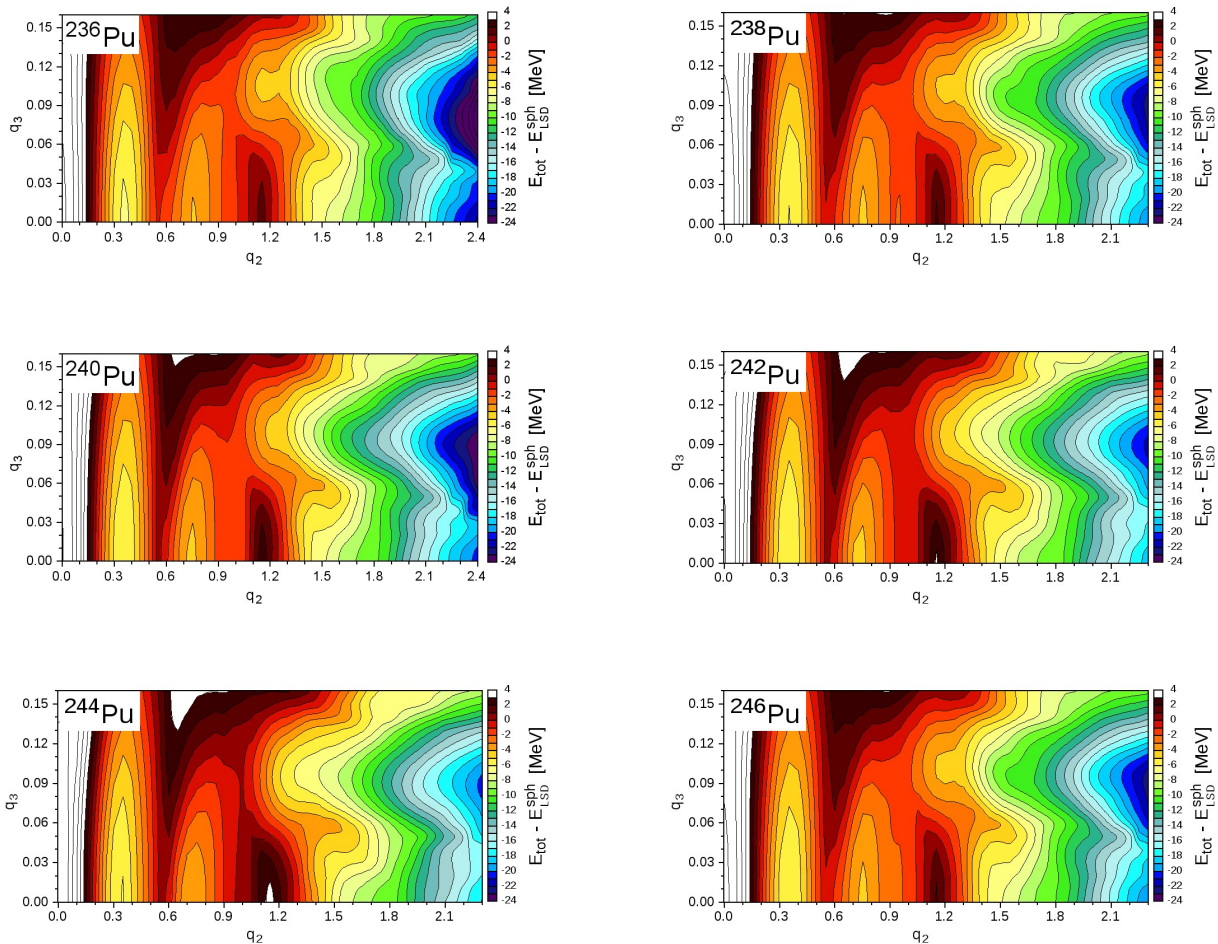

Figure 1. Potential energy surfaces on the $\left(q_{2}, q_{3}\right)$ plane of even-even $\mathrm{Pu}$ isotopes minimized with respect to $q_{4}$.

Here $u_{n E}\left(q_{2}\right)$ is the wave function for the fission (elongation) mode and $\varphi_{n}$ are the eigenfunctions of the Hamiltonian which describe the collective motion in the perpendicular directions. In the following we shall use the WKB approximation for the $u_{n E}\left(q_{2}\right)$ wave function and consider only the lowest energy eigenstate $\varphi_{n=0}$ since we are interested in fission at very low excitation energy. The effect of taking into account higher states was discussed in Ref. [11].

The probability of finding the system, for a given elongation $q_{2}$, in a deformation defined by $\left(q_{3}, q_{4}\right)$ is equal to

$$
W(q 3, q 4 ; q 2)=\left|\Psi\left(q_{3}, q_{4} ; q_{2}\right)\right|^{2}=\left|\varphi_{0}\left(q_{3}, q_{4} ; q_{2}\right)\right|^{2}
$$

Our model is still simplified further. Instead of the square of the collective wave function (3), we take the following Wigner function:

$$
W(q 3, q 4 ; q 2) \sim \exp \left\{-\frac{V\left(q_{3}, q_{4} ; q_{2}\right)-V_{\mathrm{eq}}\left(q_{2}\right)}{E_{0}+T}\right\}
$$

where $V_{\text {eq }}\left(q_{2}\right)$ is the potential minimum for a given $q_{2}, E_{0}$ is the zero-point energy, treated here as a free parameter and $T$ is the temperature of the nucleus.

The probability distribution integrated over $q_{4}$

$$
w\left(q_{3} ; q_{2}\right)=\int W\left(q_{3}, q_{4} ; q_{2}\right) d q_{4}
$$


is directly related to the fragment mass yield at given elongation $q_{2}$.

It is obvious that the fission probability should also depend on the neck radius. Following Ref. [12] we assume a neck-rupture probability $P$ in the form:

$$
P\left(q_{2}, q_{3}, q_{4}\right)=\frac{k_{0}}{k} P_{\text {neck }}\left(R_{\text {neck }}\right),
$$

where $k$ is the momentum in the direction towards fission (or simply the velocity along $q_{2}$ ), while $R_{\text {neck }}\left(q_{2}, q_{3}, q_{4}\right)$ is the deformation-dependent neck radius. $k_{0}$ plays the role of a scaling parameter. The neck rupture probability [12] can be taken e.g. in one of the following forms:

$$
P_{\text {neck }}\left(R_{\text {neck }}\right)=\left\{\begin{array}{lr}
\exp \left[-\ln 2\left(R_{\text {neck }} / d\right)^{2}\right] & \text { Gauss } \\
1 / \cosh \left[(2+\sqrt{3}) R_{\text {neck }} / d\right] & 1 / \text { cosh } \\
1 /\left[1+\left(R_{\text {neck }} / d\right)^{2}\right] & \text { Lorentz }
\end{array}\right.
$$

The parameter $d$ is the half-width of the probability and is treated here as a free adjustable parameter.

The momentum $k$ in Eq. (6) has to ensure that the probability depends on the time in which one crosses the subsequent interval in $q_{2}: \Delta t=\Delta q_{2} / v\left(q_{2}\right)$, where

$$
v\left(q_{2}\right)=\hbar k / \bar{M}\left(q_{2}\right)
$$

is the velocity towards fission. The inertia $\bar{M}\left(q_{2}\right)$ is evaluated using the approximation proposed in Ref. [21]:

$$
\bar{M}\left(q_{2}\right)=\mu\left[1+11.5 \cdot\left(B_{\mathrm{irr}} / \mu-1\right)\right]\left(\frac{\partial R_{12}}{\partial q_{2}}\right)^{2},
$$

where $B_{\text {irr }}$ is the irrotational inertia corresponding to the distance $R_{12}$ between the fragments and $\mu$ is the reduced mass. The value of $k$ in Eq. (8) depends on the difference $E-V\left(q_{2}\right)$ and on the part of the collective energy which is converted into heat $Q$ :

$$
\frac{\hbar^{2} k^{2}}{2 \bar{M}\left(q_{2}\right)}=E_{k i n}=E-Q-V\left(q_{2}\right) .
$$

In order to introduce the neck-rupture probability $P$, in our formalism one has to rewrite the probability distribution $w\left(q_{3} ; q_{2}\right)$ in the form

$$
w\left(q_{3} ; q_{2}\right)=\int W(q 3, q 4 ; q 2) P\left(q_{3}, q_{4}, q_{2}\right) d q_{4} .
$$

Such an approximation means that the fission process is spread over some region of $q_{2}$ and that for a given $q_{2}$, at fixed mass asymmetry, one has to take into account the probability to fission at a previous $q_{2}$ value, i.e. one has to replace $w\left(q_{3} ; q_{2}\right)$ by

$$
w^{\prime}\left(q_{3} ; q_{2}\right)=w\left(q_{3} ; q_{2}\right) \frac{1-\int_{q_{2}^{\prime} \leq q_{2}} w\left(q_{3} ; q_{2}^{\prime}\right) d q_{2}^{\prime}}{\int w\left(q_{3} ; q_{2}^{\prime}\right) d q_{2}^{\prime}} .
$$

The integral mass yield can then be written as the sum of all partial yields at different $q_{2}$ :

$$
Y\left(q_{3}\right)=\int w^{\prime}\left(q_{3} ; q_{2}\right) d q_{2} / \int w^{\prime}\left(q_{3} ; q_{2}\right) d q_{2} d q_{3} .
$$

As one notices from (13), the scaling factor $k_{0}$ in the expression for $P$, Eq. (6), has vanished and does no longer appear in the definition of the mass yield. Our model thus has only two adjustable parameters, $E_{0}$ in Eq. (4) and the width parameter $d$, that appears in the neck-rupture probability (7). 


\section{Results}

The potential energy surfaces (PES), relative to the corresponding spherical LSD energy, were calculated for even-even Plutonium isotopes ${ }^{236-246} \mathrm{Pu}$ in the 3D $\left(q_{2}, q_{3}, q_{4}\right)$ deformation space, where $q_{2}$ describes the elongation of the nucleus, $q_{3}$ its reflection asymmetry, and $q_{4}$ controls the neck size [8,9]. The $\left(q_{2}, q_{3} ; q_{4}^{\mathrm{min}}\right)$ cross-sections of the PES minimized with respect to $q_{4}$ are presented in Fig. 1 . Pronounced mass asymmetric $\left(q_{3} \neq 0\right)$ fission valleys are visible in all isotopes beyond $q_{2} \approx 1$. Symmetric fission valleys are generally present but lay always higher by a few $\mathrm{MeV}$, and with a substantial ridge separating them from the asymmetric valleys.

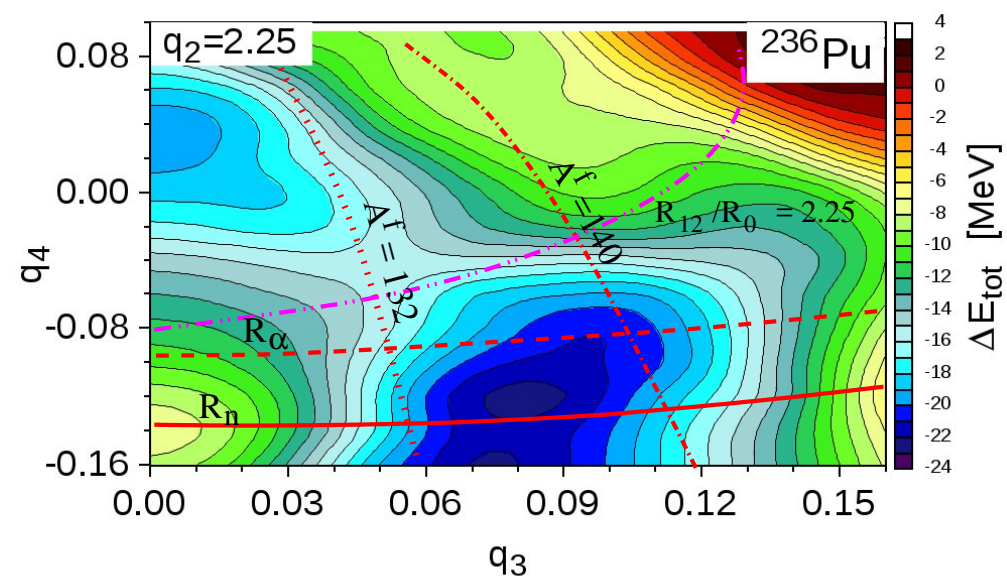

Figure 2. $\left(q_{3}, q_{4}\right)$ PES cross-section of ${ }^{236} \mathrm{Pu}$ for $q_{2}=2.25$.

It is obvious that 2D maps do not give a complete information about the way in which a system goes to fission. The neck dimension, which for a given elongation $q_{2}$ and asymmetry $q_{3}$ depends on $q_{4}$, decides when fission occurs. The map of the PES of ${ }^{236} \mathrm{Pu}$ on the $\left(q_{3}, q_{4}\right)$ plane for $q_{2}=2.25$ is presented as an example in Fig. 2. Lines corresponding to a neck radius equal to the one $\left(R_{n}\right)$ of a nucleon, an alpha-particle $\left(R_{\alpha}\right)$, as well as of the heavier mass fragment $A_{h}^{f}=132$ and $A_{h}^{f}=140$ are marked in the plot. It is seen that the deformation parameter $q_{3}$ governs mostly the fragment mass, while $q_{4}$ is related to the neck size. The line corresponding to the distance $R_{12} / R_{0}=2.25$ between the fragment mass centres is also shown. Note that in all other points of the map $R_{12}$ differs from this mean value by less than 5\% what proves that at large elongations $q_{2}$ is roughly a measure of the distance between the fragments.

More informative are the projections of the PES's for different elongations $q_{2}$ onto the $\left(A_{h}^{f}, R_{\text {neck }}\right)$ maps presented in Fig. 3 for ${ }^{236} \mathrm{Pu}$. It is seen that the structure of the bottom of the fission valley changes with increasing elongation of the nucleus. At smaller deformations $q_{2} \leq 2.25$, the bottom of the valley in the vicinity of $R_{\text {neck }}=R_{n}$, the size of a nucleon (thin solid), corresponds to the mass of the heavier fragment $A_{h}^{f}=140$. At $q_{2}=2.3$ already two valleys at 138 and 144 are visible, while at $q_{2}=2.35$ three fission valleys corresponding to 136,141 and 146 are formed. The sizeable dependence of the PES in the $\left(A_{h}^{f}, R_{\text {neck }}\right)$ plane on the elongation coordinate $q_{2}$ shows how important is to take into account the subsequent steps in the fission process as explained above. 

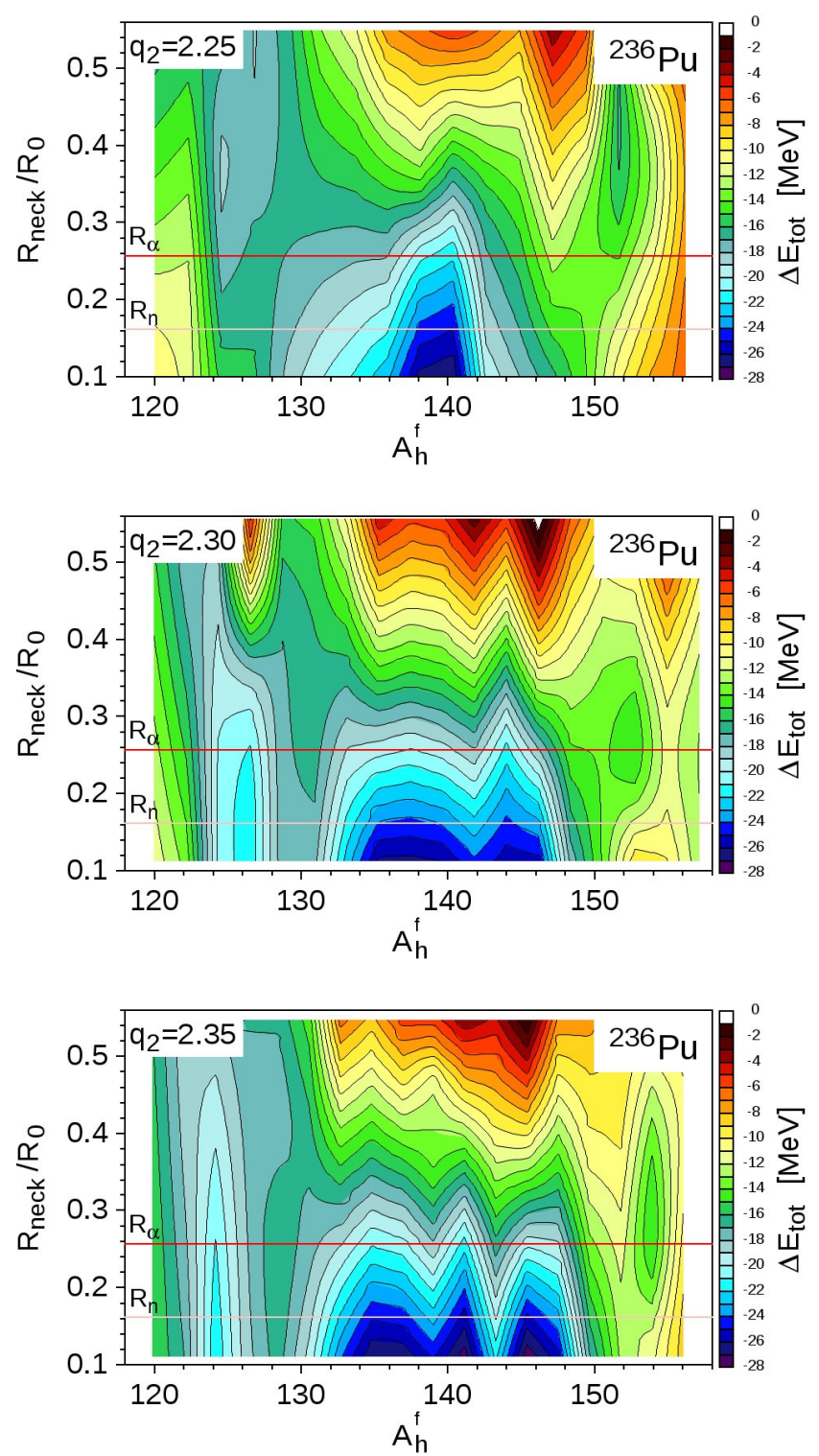

Figure 3. Potential energy surfaces for different elongations $q_{2}$ projected onto the $\left(A_{h}^{f}, R_{\text {neck }}\right)$ plane.

The Wigner function (5) integrated over $q_{4}$ is presented in Fig. 4 for different elongations $q_{2}$ and projected onto the $A_{h}^{f}$ axis, instead of the asymmetry parameter $q_{3}$. Its maximum changes accordingly to the properties of the fission valleys shown in Fig. 3. This set of Wigner functions weighted with the neck-size dependent fission probability gives access to the mass yields (11) for different elongations $q_{2}$.

The final heavy-fragment mass yield (13), obtained for three different neck-breaking probabilities (7) is shown in Fig. 5 for ${ }^{240} \mathrm{Pu}$, and compared to the yield measured in spontaneous fission [19]. The differences between the theoretical yields are small as can be seen in the figure. We shall therefore present in the following the results obtained with the Gauss distribution only keeping the half-width fixed at $d / R_{0}=0.15$ which roughly corresponds to the nucleon radius. The width-parameter $E_{0}$ of the 


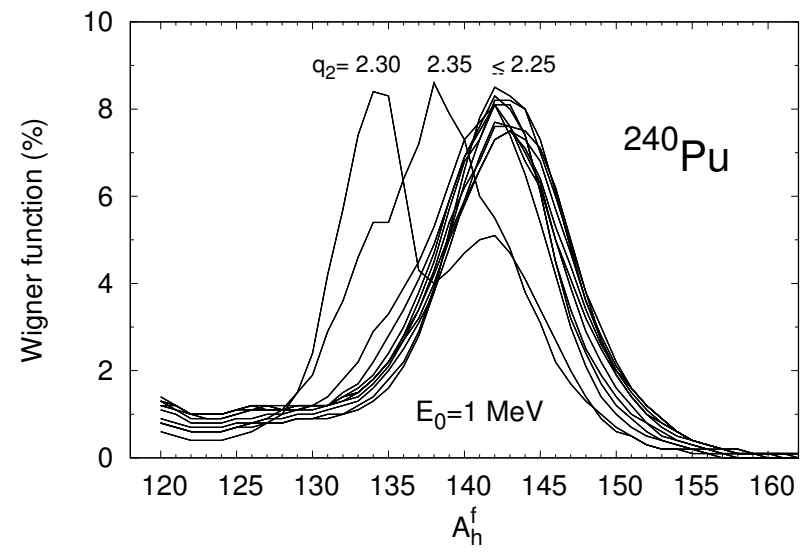

Figure 4. Wigner function for different elongations $q_{2}$ as a function of the heavy-fragment mass number $A_{h}^{f}$.

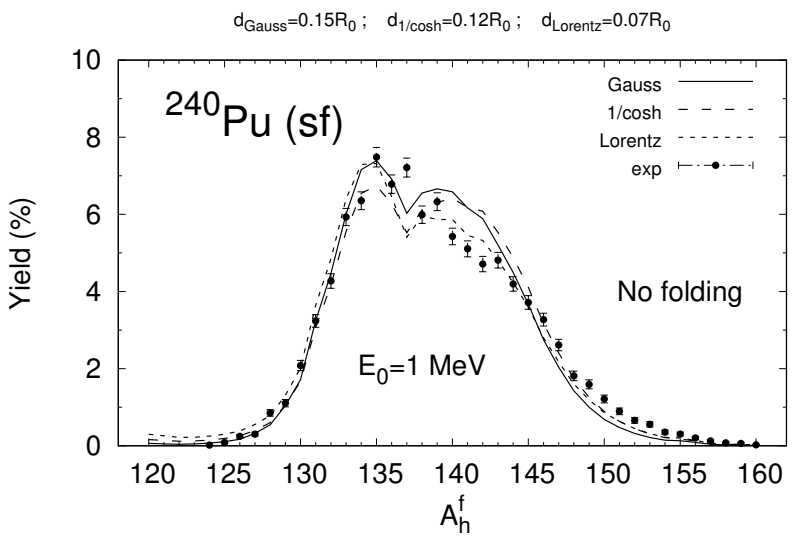

Figure 5. Heavy fragment mass yield for fission of ${ }^{240} \mathrm{Pu}$ as obtained with three different neck-breaking probability functions (7). The half-width of each function is written above the plot. The calculation is compared to the yield measured in spontaneous fission in Ref. [19].

Wigner function was taken as $E_{0}=1 \mathrm{MeV}$ what corresponds to a typical energy of collective modes in two dimensions ( $q_{3}$ and $\left.q_{4}\right)$.

The spontaneous fission yields for six even-even Pu isotopes are compared in Fig. 6 with the experimental data whenever available [19]. Please note that these theoretical estimates were obtained with only two adjustable parameters $d / R_{0}=0.15$ and $E_{0}=1 \mathrm{MeV}$. All other parameters of the model are unchanged as compared to those adjusted years ago when considering still different data.

The total kinetic energy (TKE) of the fragments is evaluated in the point-charge approximation of the Coulomb interaction energy between the fragments. No pre-fission kinetic energy is used in the present calculation, i.e. we assume that the whole energy gain on the slope to scission is transferred into heat. One has to bear in mind that for a given elongation $q_{2}$ the mass of the fragment $A_{h}^{f}\left(q_{3}, q_{4} ; q_{2}\right)$ and the $\operatorname{TKE}\left(q_{3}, q_{4} ; q_{2}\right)$ are functions of $q_{3}$ and $q_{4}$. So, it is easy to map the yields from the $\left(q_{3}, q_{4}\right)$ onto the (TKE, $A_{h}^{f}$ ) plane. The experimental data have a finite resolution in mass and in TKE, which in not negligible for the present cases [19]. Typical values are $\sigma_{\mathrm{TKE}}=10 \mathrm{MeV}$ and a variance of $\sigma_{A_{h}^{f}}=1.5$ a.m.u. Hence, the theoretical $A_{h}^{f}$ and TKE yields have to be folded according to these values before they can be compared to the experiment. The resulting folded TKE yield is presented in Fig. 7 (top). 

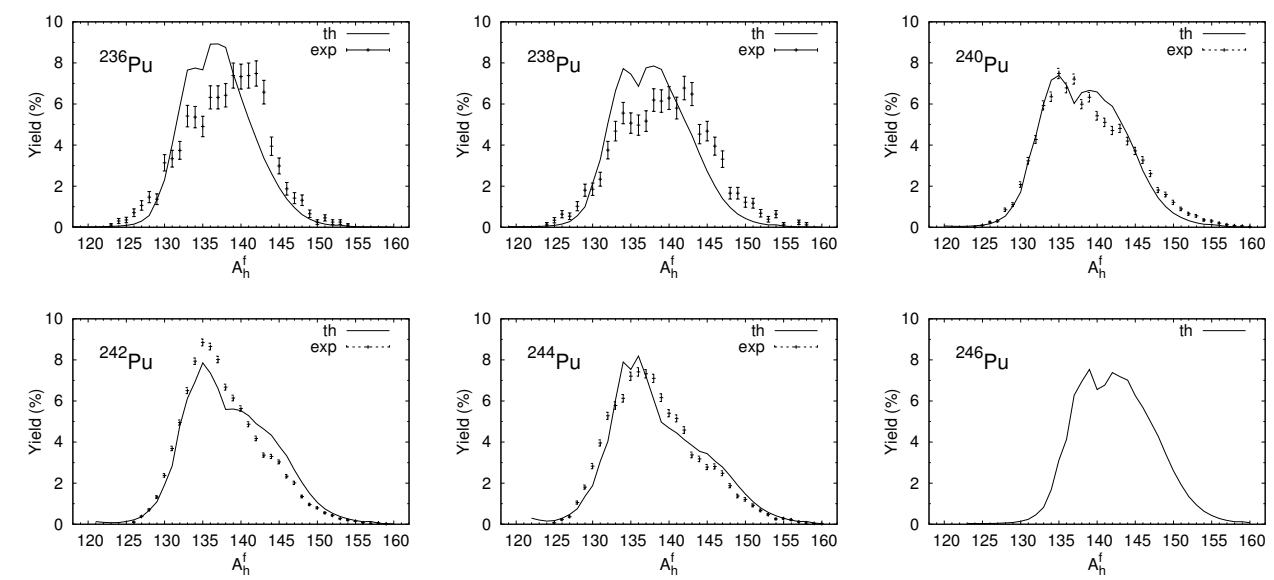

Figure 6. Same as in Fig. 5 for fission of ${ }^{236-246} \mathrm{Pu}$ but for the optimal calculation (see the text). The theoretical results, obtained here without any folding procedure, are compared to the yield measured in spontaneous fission in Ref. [19]) for ${ }^{236-244} \mathrm{Pu}$.

One can see in the figure that the maximum of the TKE yield for ${ }^{240} \mathrm{Pu}$ corresponds to TKE $\approx 179 \mathrm{MeV}$ what is in line with the measured data [20]. The calculated correlation between the heavy-fragment mass and the TKE is presented in Fig. 7 (bottom). Although a quantitative analysis is still to be done, it is already noted that the theoretical correlation shows a trend similar to the experiment [22], with a mean TKE larger for the lighest $\left(A_{h}^{f} \approx 132\right)$ heavy-fragment group, than for the heaviest $\left(A_{h}^{f} \approx 140\right)$ group. The relatively good agreement of the TKE distribution and mean value with the experiment can be considered as quite encouraging. Keep in mind, however, that our theoretical approach is still quite crude, with the use of a Wigner functions for the probability distribution instead of the true quantum mechanical density distributions, the absence in our calculations of extremely elongated shapes, since it was impossible to construct for them a reliable Wigner function, the inclusion of only the lowest energy phonon in the direction perpendicular to the scission mode, i.e. neglecting the dynamical coupling of the fission and the perpendicular modes.

\section{Summary}

Summing up the following conclusion can be drawn from our investigation:

- an effective three-dimensional set of Fourier deformation parameters is used to describe the fission process,

- a collective 3D model describing elongation, mass asymmetry and neck modes was introduced,

- a Wigner distribution is assumed for the neck and mass asymmetry degrees of freedom,

- a neck-breaking probability depending on the neck-size has been adopted,

- our 3D model which couples fission, neck and mass asymmetry modes is able to describe the main features of the fragment mass and total kinetic energy yields. 

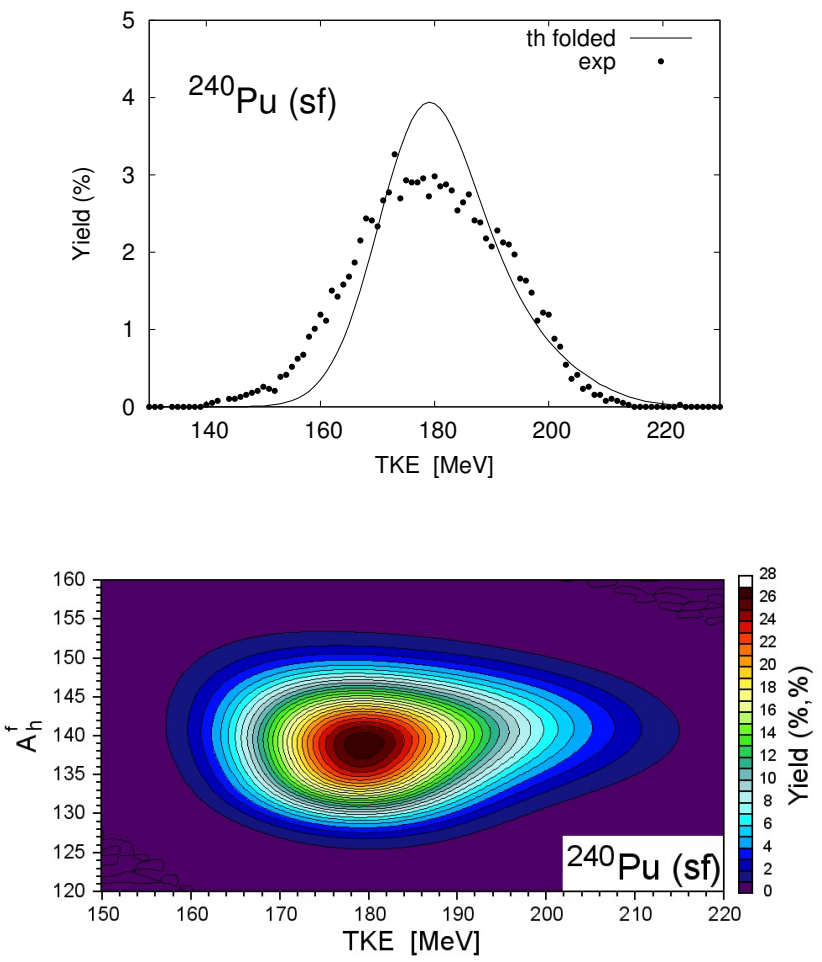

Figure 7. Top: calculated and experimental (as measured in spontaneous-fission [20]) TKE distribution for ${ }^{240} \mathrm{Pu}$. Bottom: calculated correlation between the heavy-fragment mass and the TKE. Calculated mass and TKE values were folded with the experimental resolution (see text).

This contribution presents the present status of our research. Similar calculations are going to be performed to describe low-energy fission of other isotopes. In parallel, we aim to develop a Langevin code to study fission at higher energies with the new Fourier deformations space.

This work has been partly supported by the Polish-French COPIN-IN2P3 collaboration agreement project No. 08-131 and by the Polish National Science Centre, grant No. 2013/11/B/ST2/04087.

\section{References}

[1] L. Meitner, O.R. Frisch, Nature 143, 239 (1939)

[2] N. Bohr, J.A. Wheeler, Phys. Rev. 56, 426 (1939)

[3] A. Dobrowolski, K. Pomorski, J. Bartel, Phys. Rev. C 75, 024613 (2007)

[4] J.R. Nix, Nucl. Phys. A 130, 241 (1969)

[5] M. Brack, J. Damgaard, A.S. Jensen, H.C. Pauli, V.M. Strutinsky, and C.Y. Wong, Rev. Mod. Phys. 44, 320 (1972)

[6] J. Bartel, B. Nerlo-Pomorska, K. Pomorski, and C. Schmitt, Phys. Scr. 89, 054003 (2014)

[7] V.V. Pashkevich, Nucl. Phys. A 169, 275 (1971)

[8] K. Pomorski, B. Nerlo Pomorska, J. Bartel, C. Schmitt, Acta. Phys. Polon. B Supl. 8, 667 (2015) 
[9] C. Schmitt, B. Nerlo Pomorska, K. Pomorski, J. Bartel, Phys. Rev. C 17, 034612 (2017)

[10] S.G. Nilsson et al., Nucl. Phys. A 131, 1 (1969)

[11] B. Nerlo-Pomorska, K. Pomorski and F.A. Ivanyuk, Acta Phys. Pol. B Supl. 8, 669 (2015)

[12] K. Pomorski, B. Nerlo-Pomorska and F.A. Ivanyuk, Eur. Phys. J. A 53, 59 (2017)

[13] K. Pomorski and J. Dudek, Phys. Rev. C 67, 044316 (2003)

[14] K.T.R. Davies and J.R. Nix, Phys. Rev. C 14, 1977 (1976)

[15] A. Dobrowolski, K. Pomorski, and J. Bartel, Comp. Phys. Comm. 199, 118 (2016)

[16] V.M. Strutinsky, Yad. Fiz. 3, 614 (1966); Nucl. Phys. A 95, 420 (1967)

[17] A. Góźdź and K. Pomorski, Nucl. Phys. A 451, 1 (1986)

[18] S. Pilat, K. Pomorski, A. Staszczak, Zeit. Physik A 332, 259 (1989)

[19] L. Dématté et al, Nucl. Phys. A 617, 331 (1997)

[20] C. Wagemans, E. Allaert, A. Deruytter, R. Barthelemy, and P. Schillebeeckx, Phys. Rev. C 30, 218 (1984)

[21] J. Randrup et al., Phys. Rev. C 13, 229 (1976)

[22] P. Schillebeeckx, C. Wagemans, A.J. Deruytter, and R. Barthelemy, Nucl. Phys. A 545, 623 (1992), and S. Oberstedt, private communication.

\section{Appendix}

A new nuclear shape parametrization $[8,9]$ is used that gives an expansion of the nuclear surface in the form of a Fourier analysis in cylindrical coordinates

$$
\frac{\rho_{s}^{2}(z)}{R_{0}^{2}}=\sum_{n=1}^{\infty}\left[a_{2 n} \cos \left(\frac{(2 n-1) \pi}{2} \frac{z-z_{\mathrm{sh}}}{z_{0}}\right)+a_{2 n+1} \sin \left(\frac{2 n \pi}{2} \frac{z-z_{\mathrm{sh}}}{z_{0}}\right)\right],
$$

where, similarly to the famous "Funny-Hills" (FH) shape parametrization [5], $\rho_{s}(z)$ defines the distance of the equivalent sharp surface from the symmetry $z$ axis, and $z_{0}$ is half the elongation of the nuclear shape along that axis with end points located at $z_{\min }=z_{\mathrm{sh}}-z_{0}$ and $z_{\max }=z_{\mathrm{sh}}+z_{0}$. The coordinate $z_{\mathrm{sh}}$ is chosen so as to locate the centre of mass of the shape at the origin of the coordinate system. $R_{0}$ represents the radius of the corresponding spherical shape having the same volume and $z_{0}=c R_{0}$.

The parameters $a_{2}, a_{3}, a_{4}$ are related to elongation, left-right asymmetry, and neck degree of freedom, respectively. More and more elongated prolate shapes correspond to decreasing values of $a_{2}$, while oblate ones are described by $a_{2}$ larger than one, which is somehow contrary to the traditional definition of a quadrupole deformation parameter. So, it was proposed in Ref. [9] to use a more natural definition of deformation parameters which in addition ensures that along the LD fission path these parameters show only a small variation around zero:

$$
\begin{aligned}
& q_{2}=\frac{a_{2}^{(0)}}{a_{2}}-\frac{a_{2}}{a_{2}^{(0)}}, \quad q_{3}=a_{3}, \quad q_{4}=a_{4}+\sqrt{\left(\frac{q_{2}}{9}\right)^{2}+\left(a_{4}^{(0)}\right)^{2}}, \\
& q_{5}=a_{5}-\left(q_{2}-2\right) \frac{a_{3}}{10}, \quad q_{6}=a_{6}-\sqrt{\left(\frac{q_{2}}{100}\right)^{2}+\left(a_{6}^{(0)}\right)^{2}} .
\end{aligned}
$$

Here $a_{n}^{(0)}$ stands for the value of the $a_{n}$ coefficient in the spherically symmetric case:

$a_{2}^{(0)}=1.03205, a_{4}^{(0)}=-0.03822, a_{6}^{(0)}=0.00826$. 